Session 2220

\title{
ROLE OF A MATLAB REAL-TIME HARDWARE INTERFACE WITHIN A SYSTEMS MODELING COURSE
}

\author{
Joel M. Esposito, Matthew G. Feemster, and John M. Watkins \\ United States Naval Academy \\ Weapons \& Systems Engineering Department, Mail Stop 14A, \\ 105 Maryland Ave., Annapolis, MD 21402-5034 \\ E-mail: esposito, feemster, watkins@usna.edu
}

\begin{abstract}
In an effort to offer students a more tangible understanding of system modeling concepts, the Quanser/MATLAB hardware interface has been employed at USNA in the laboratory setting of an undergraduate modeling course. Specifically, the SIMULINK-based interface has enabled students to model various physical systems and then compare the system performance predicted by their simulation to the actual response of the physical system. In this paper, an actual case study performed by the midshipmen utilizing the Quanser interface system with a rigid-link, flexible-joint robotic manipulator is presented.
\end{abstract}

\section{Introduction}

The ability to construct accurate mathematical models, such as transfer functions and state space representations, of complex dynamic systems is the corner stone for classical control development and analysis. That is without a suitable model of a physical plant, many of the classical approaches for compensator design and stability analysis are rendered ineffective. While the discipline of modeling is deeply rooted in physics, the process of constructing representative models can often be as much of an art as a science. Identifying system parameters, utilizing simplifying assumptions, and judging the validity of the resulting simulation results are very difficult topics to convey solely in a lecture format. Typically, algorithms and/or textbook procedures cannot serve as alternatives for the engineering intuition garnered through hands-on laboratory experience. One of the most crucial steps in teaching the flow of the modeling procedure (see Figure 1) to undergraduate engineering students is the "validation" of their developed model. In order to fully validate the system model, an experimental test must be performed on the target plant with all pertinent data collected and stored for comparison to the corresponding simulation results. Without such feedback it is extremely difficult to comprehend the impact of modeling assumptions, to observe measurement errors, or to locate possible modeling mistakes. Due to this need, a hardware interface system must be utilized that can interact with the physical plant and sensors such that the experimental test can be realized. Currently, we are employing the WinCon real-time interface with SIMULINK for Quanser Inc. 
operating under Windows 2000 operating system; however, other hardware interface alternatives have been successfully employed at the undergraduate level ${ }^{1}$.

In this paper, we describe our attempt at designing a series of laboratory exercises for a junior-level undergraduate modeling course taught at the United States Naval Academy during the fall 2003 semester. The exercises required the students to develop a state space representation of a rigid-link, flexible joint robotic manipulator actuated by a DC motor, identify system parameter values of the actual system, evaluate the simulation vs. experiment results, critique the proposed simulation model, and then augment the model in an effort to improve simulation accuracy. In each component of the project, the students utilized the WinCon real-time interface to actuate the robotic link and to collect measurements from the numerous sensors embedded on the physical plant. In Section 2, we provide a brief overview of the course objectives. In Section 3, a description of the WinCon realtime interface and the rigid-link, flexible joint system is presented. The modular laboratory exercises are described in Section 4. In Section 5, the success of the sequence of laboratory exercises is quantified in the form of follow-up evaluation sheets completed by the participating students.

\section{Course Overview}

ES301, "Dynamic Systems Modeling and Simulation," which is a three credit hour course offered by the United States Naval Academy's Systems Engineering Department, served as one test-bed for the integration of the hardware interface system. The Systems Engineering major has at its core a feedback control system focus, with opportunities for students to take upper level courses in several application areas including robotics and embedded control systems. ES301 is required for all students in the major and is typically taken during the junior year. The objectives of the course are to have students be proficient in constructing mathematical models, such as transfer functions and state space models, of various physical systems, such as mechanical, electrical and hydraulic systems. In addition topics such as numerical integration, system identification, and the use of MATLAB and SIMULINK tools within the modeling context are covered. Prior student exposure to MATLAB and SIMULINK is at an introductory level.

Traditionally the focus of the laboratory portion of the course has been a mixture of SIMULINK exercises and hardware

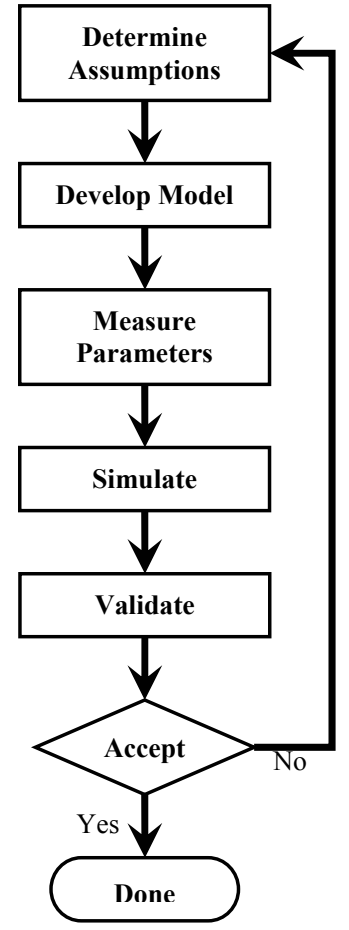

Figure 1: The Modeling Process demonstrations. Typically, students would attempt to model a system on paper, and then construct a SIMULINK diagram to predict the behavior of the plant. A demonstration may have been conducted to allow them to observe how the physical system's evolution corresponds to their predictions; however, the comparison was strictly qualitative. Recently the course was revised to place greater emphasis on first principles and to develop more physical intuition about engineering systems. To this end, we have designed a series of exercises that extensively utilize the WinCon hardware interface. 


\section{Equipment}

The laboratory experiments have been developed with equipment from Quanser, Incorporated serving as the core. The experimental environment incorporates a physical plant, analog and digital sensors, $\mathrm{A} / \mathrm{D}$ and $\mathrm{D} / \mathrm{A}$ converters, and the WinCon real-time interface with SIMULINK. The experimental apparatus permits the students to concentrate on the modeling and requires the students to address the interfacing issues only at a high level. An overview of the experimental environment is shown in Figure 2. A power module manages the flow of power and information to and from the DC motor. The power module also contains the power electronics required to drive the DC motor. A terminal board connects the WinCon software interface to the hardware. The I/O board is equipped with sixteen $\mathrm{A} / \mathrm{D}$ channels, four $\mathrm{D} / \mathrm{A}$ channels, six encoder or digital $\mathrm{I} / \mathrm{O}$ channels ${ }^{2}$.

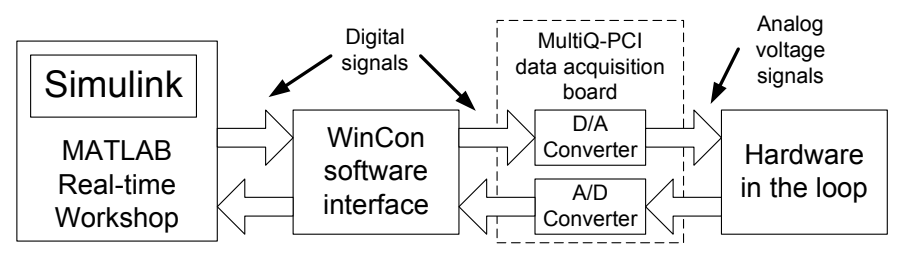

Figure 2: Experimental Environment

The basic physical plant is the DC motor shown in Figure 3. The motor is equipped with a potentiometer and a digital encoder to measure the angular position of the output shaft and a tachometer to measure the angular velocity of the output shaft. As shown in Figure 4, the motor shaft is rigidly coupled to the hub of the robotic link. This hub is coupled to a robotic arm through a spring-loaded rotary flexible joint. The angle of the arm relative to the hub is measured via a digital encoder. The entire arrangement is referred to as the flexible joint robotic $\mathrm{arm}^{3}$.

Obviously, it is not standard practice to connect robotic arms with springs. However, all joints have some compliance and this compliance can be approximated as a rotational spring. In a typical industrial robot however, the stiffness of the rotational spring is sufficient to have minimal impact on performance and stability. However, in space applications, such as the robotic arm attached to the space shuttle, the stiffness of the rotational spring is very light. This is due to the long length (and consequently large inertia) of the arm and the succession of gear stages required to drive this large inertia ${ }^{4}$. 


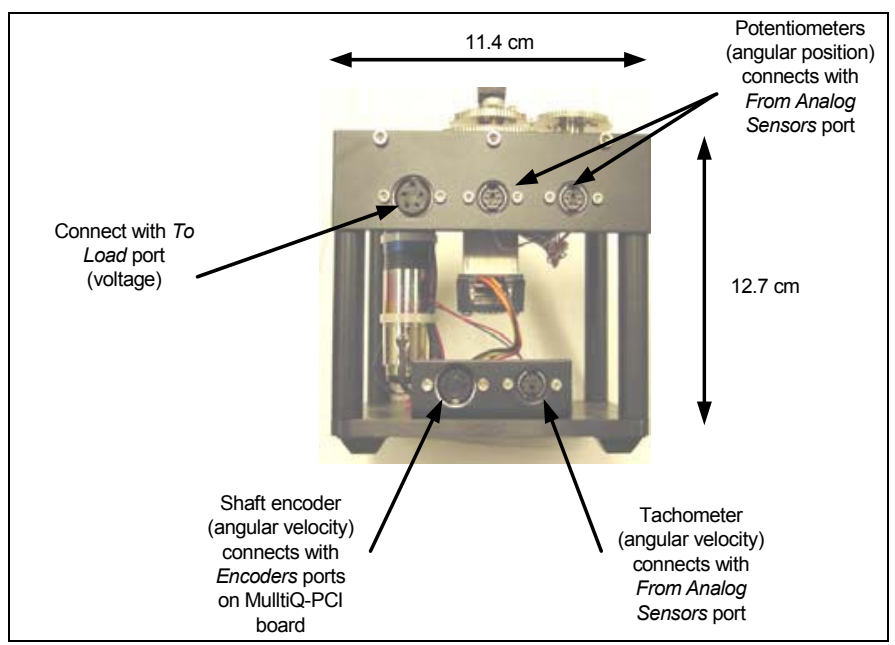

Figure 3: Quanser DC Motor

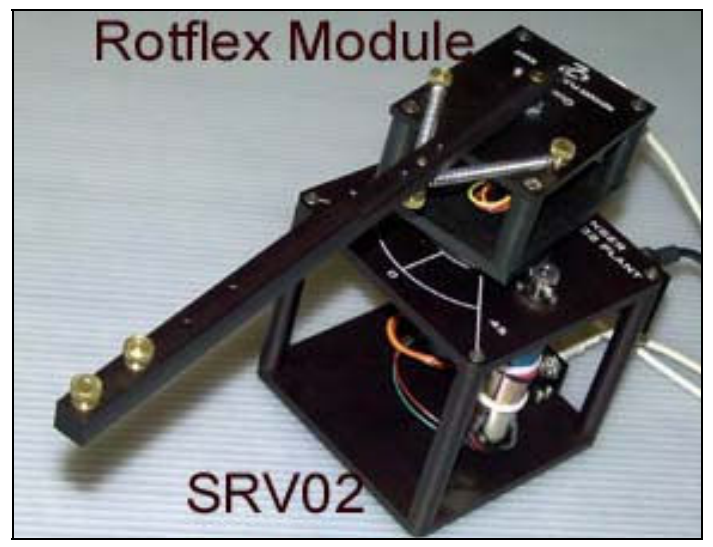

Figure 4: Flexible Joint Robotic Arm

The most attractive feature of the experimental apparatus is the SIMULINK interface. Figure 5 shows an example of the SIMULINK diagram for measuring the DC motor response to a step change in the input voltage. The SIMULINK diagram serves as a high-level bock diagram of the system. The WinCon and MATLAB real-time software have special blocks to connect to the A/D and D/A converters and the optical encoders. Standard SIMULINK blocks are used to create the rest of the system diagram. As a result, there are few software limitations on the structure of the experiment.
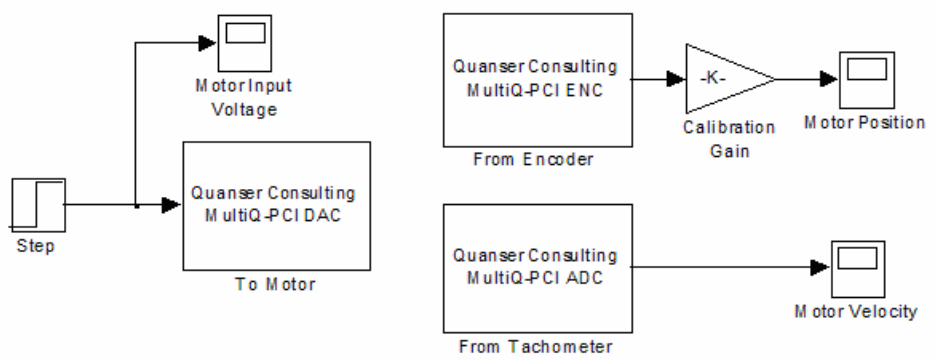

Figure 5: SIMULINK Diagram for DC Motor Experiment 


\section{Laboratory Exercise}

The ultimate goal of the laboratory exercise was to construct and verify a state space representation of the flexible robotic link actuated by a DC motor (see Figure 4). A modular approach to modeling the system was employed as opposed to a lumped system development to allow for individual parameter identification (e.g., identification of the motor torque constant of the DC motor, passive damping constant of the robotic link, etc.) of each respective system that would be otherwise difficult to derive due to the resulting coupling of system parameters. In essence, a separate analysis was performed on the flexible joint robotic arm and the DC motor system in an effort to completely identify all pertinent subsystem parameters. Once each subsystem is completely identified, the state space representation for the coupled system is then derived. Upon completion of development of the state space model, the midshipmen were then required to validate their model against experimental measurements. If discrepancies existed between the simulation and the experiment, then the proposed model was to be critiqued and adjusted to improve accuracy.

\subsection{Modeling of the Flexible Robotic Link}

In the first exercise, which took place over two two-hour lab periods, the students modeled only the flexible link subsystem. The emphasis was on devising creative experiments to determine the various system parameters such as the spring constant $K_{l}$, damping constant $\beta_{l}$, and the link inertia $J_{l}$. Students were given a one page handout stating various ground rules and a rough outline of what was expected of them, but no specific procedures were provided. The students were asked to perform the following tasks:

1. Draw a schematic of the system. Be sure to indicate any assumptions or approximations.

2. Create a mathematical model of the system (i.e., a set of differential equations).

3. Determine what physical parameters are needed for the model-including any initial conditions. Determine which state measurements are required and/or devise a set of experiments to determine them.

4. Create a SIMULINK simulation diagram of the proposed model.

5. Experimentally determine the system response and compare it to a simulation produced using the same initial conditions.

6. Reflect on the accuracy of your model, and any causes of error. Return to step 1 if the results were not satisfactory.

The midshipmen were also provided with a sheet describing how to collect data from the Quanser interface system. Connection and configuration of the data acquisition equipment and software was done in advance of the laboratory period by the instructors in order to keep the focus of the students on the modeling exercise.

Remark \#1 Step 1 required significant instructor assistance since there are several ways to model the flexible joint system that are not obvious. The instructors recommended that the students model the two linear springs at the base of the link as a single rotational spring. It was also recommended that the students model the friction in the bearings as a rotational damper in order to preserve the linearity of the model. 
Remark \#2 Step 2 proved to be an easy task for the midshipmen due to the correlation with the classroom lecture. The final equation of motion for the rigid link flexible joint system is given by the following

$$
J_{l} \ddot{\theta}_{l}=-\beta_{l} \dot{\theta}_{l}-K \theta_{l}+\tau_{l}
$$

where $\tau_{l}$ represents any applied torque to the link.

Remark \#3 The students devised many of the measurements in Step 3 autonomously such as measuring the mass and length of the bar to calculate its moment of inertia $J_{l}$ or measuring the spring constant, $K_{l}$, by measuring the joint's angular deflection under its own weight (when laid on its side). The principle complication in this exercise lie in determining the damping constant, $\beta_{l}$. Because the system is very lightly damped and the true bearing friction is nonlinear, it was very difficult to design an experiment to measure this quantity. Identifying this parameter required significant guidance provided via a handout. Figure 6 displays typical data taken after displacing the arm about 10 degrees from the equilibrium position and then releasing. The suggested method to compute the damping constant is to measure the amplitude of the vibration at two different oscillations, spaced about 10 cycles apart. If $x_{1}$ is the amplitude of the first oscillation and $x_{N}$ is the amplitude of the $N^{\text {th }}$ oscillation, the damping ratio can be estimated as follows

$$
\zeta=\frac{\ln \left(x_{1} / x_{N}\right)}{2 N \pi}
$$

The damping ratio $\zeta$ can be related to $\beta_{l}$ by equating the Laplace transform of the standard second order system with the Laplace transformed model.

$$
\frac{A \omega_{n}^{2}}{s^{2}+2 \omega_{n} \zeta s+\omega_{n}^{2}}=\frac{1 / J_{l}}{s^{2}+\left(\beta_{l} / J_{l}\right) s+\left(K_{l} / J_{l}\right)}
$$

However, the calculation is highly sensitive to measurement errors and fundamentally assumes that the system is linear - which it is not. 


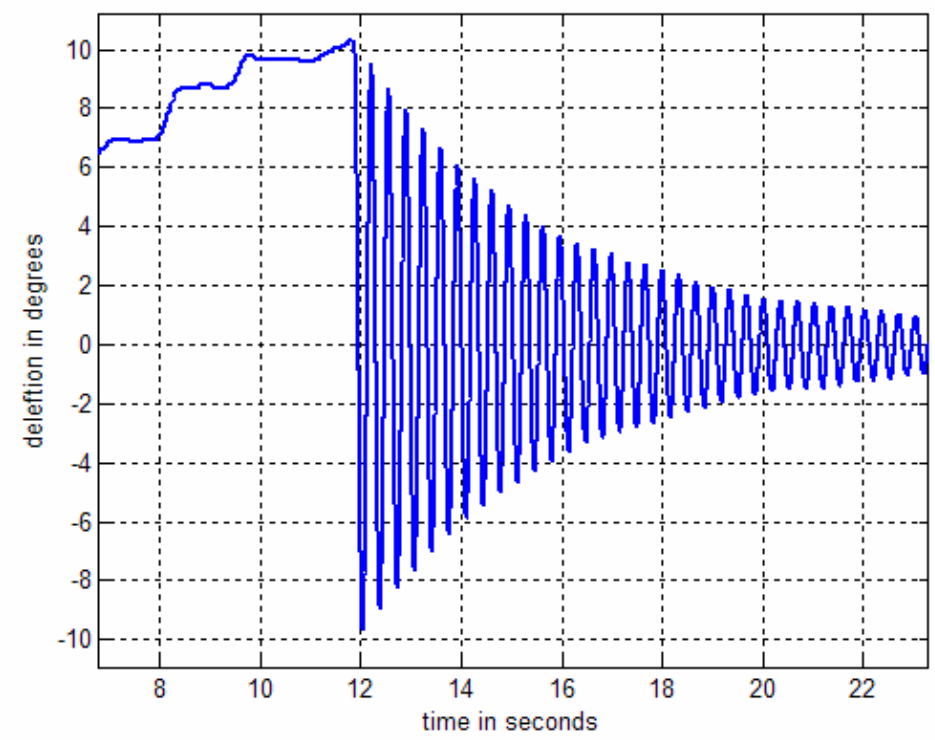

Figure 6: Impulse Response of Robotic Link

Remark \#4 Most students completed steps 4, 5, and 6 with relative ease. The student's simulations were typically consistent with the experiments and quantitatively accurate for the first few oscillations. However, the nonlinear friction effects eventually created a divergence resulting in the deviation of the simulation trace from the measured arm angle. Several students realized there were errors present in their models after comparing the experimental and simulation results.

\subsection{Modeling of the DC Motor}

The DC motor modeling portion of the overall exercise was conducted over one, two-hour laboratory period. At the time of the laboratory, the midshipmen were being exposed to the different modeling representations of a DC motor system (i.e., transfer function and state space representations) in the classroom lectures. As a result, this exercise is more focused on the experimental identification of select parameters of the DC motor system as shown in Figure 7,

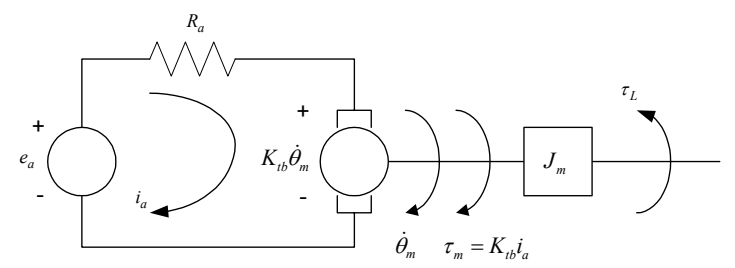

\section{Figure 7: DC Motor Representation}

where $e_{a}$ and $i_{a}$ denote the control voltage input and armature current, respectively, $R_{a}$ represents the armature resistance, $K_{t b}=K_{b}=K_{t}$ denote the motor back-e.m.f./torque constants, $J_{m}$ represents the equivalent inertia effects, $\dot{\theta}_{m}$ represents the motor output velocity, $\tau_{m}$ denotes the motor developed torque, and $\tau_{L}$ captures the loading effects. In the lab handout, the students were not 
explicitly provided with the representation of Figure 7; however, prior classroom lectures on DC motors proved sufficient to allow the students to quickly formulate the following transfer function

$$
\frac{\dot{\theta}_{m}(s)}{E_{a}(s)}=\frac{\frac{1}{K_{t b}}}{\left(\frac{R_{a} J_{m}}{K_{t b}^{2}}\right) s+1} .
$$

In order to completely identify the DC motor model of (0.4), the system parameters $R_{a}, K_{t b}$, and $J_{m}$ needed to be determined. In order to calculate the DC armature resistance $R_{a}$, a measurement of resistance across the motor terminals was performed by the students. In order calculate the remaining parameters $K_{\tau}$ and $J_{m}$, the midshipmen were required to utilize two different experimental approaches: i) the step response approach and ii) Bode frequency analysis approach.

For the step response approach, the midshipman applied a $3.0 \mathrm{~V}$ step change at $t=2.0(\mathrm{sec})$ to the motor terminals and measured, via the SIMULINK/Quanser environment, the resulting change in motor velocity (see Figure 8). From Figure 8, the midshipman then utilized the system DC gain and rise time characteristics (Note: rise time performance can be utilized since armature inductance has been neglected resulting in the first order system of (0.4)) in order to identify $K_{t b}$ and $J_{m}$, respectively.

Though the model can be identified with the step response experiment, the midshipmen were also required to obtain a Bode frequency response of the DC motor system for the range $1.0 \mathrm{rad} / \mathrm{sec}$ to $200.0 \mathrm{rad} / \mathrm{sec}$. In order to prevent a long delay in data collection for the range of frequencies, we provided the students with the custom SIMULINK/Quanser interface block in Figure 9.

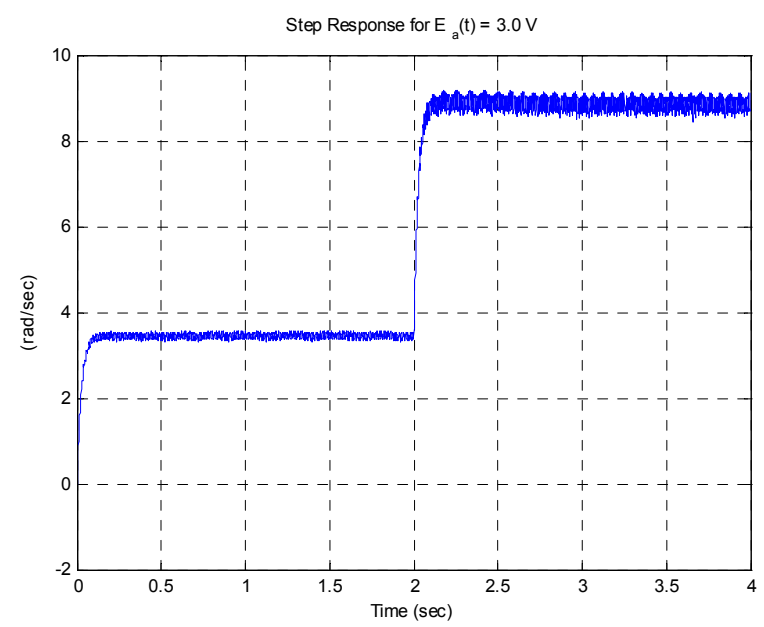

Figure 8: DC Motor Step Response 


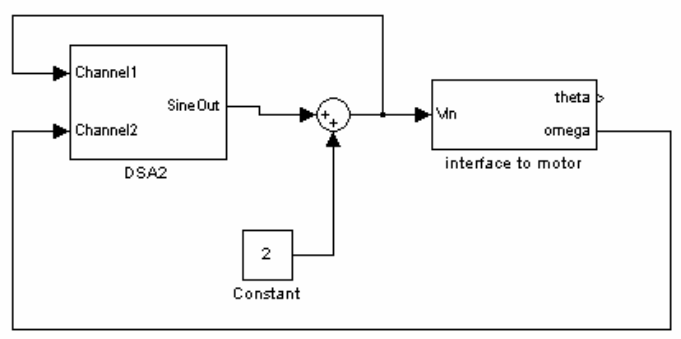

\section{Figure 9: Custom Bode Frequency Response Collection Diagram}

For the specified range of frequencies, the diagram of Figure 9 applies a sinusoidal voltage input at the specified frequency, waits for the transient effects to attenuate, and then measures and records the resulting output motor velocity. This block significantly reduces the amount of time required to collect frequency response data over the wide range of frequencies.

From Figure 10, the students then utilized the Bode magnitude at low frequencies and the corner frequency approximation to estimate the motor torque constant $K_{t b}$ and the equivalent inertia $J_{m}$, respectively.

Remark \#1 Since the desired output of the system is considered as either motor position/speed, the effects due to the armature inductance can be neglected. The midshipmen were not explicitly informed within the laboratory assignment that the DC armature inductance can be neglected; however, the time separation of the electrical system as compared to the mechanical system was reinforced within the classroom lecture.

Remark \#2 For this exercise, viscous damping effects due to such phenomena as bearing friction was neglected in the derivation of (0.4). This modeling assumption was explicitly conveyed to the midshipmen.

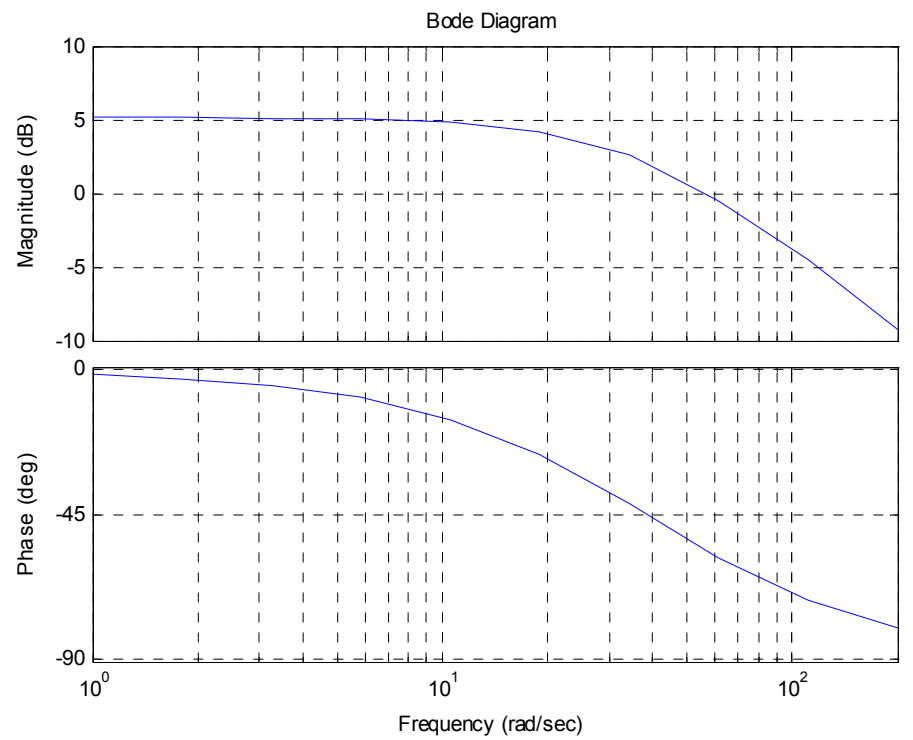




\section{Figure 10: DC Motor Frequency Response}

Remark \#3 Since the Quanser DC motor shaft is directly connected to a gear set, the angular position sensors (i.e., the optical encoder and the resistive potentiometer) that are connected to the gear set do not measure the actual DC rotor displacement. In contrast, the tachometer is directly connected to one end of the motor shaft and therefore measures the true rotor velocity. In an effort to remove potential confusion and to account for the tachometer sensor placement, we decided to select the position and velocity after the gear train as the desired output. As a result, we provided the midshipmen with a customized SIMULINK/Quanser interface block (see Figure 11) that accounted for the gear effects.

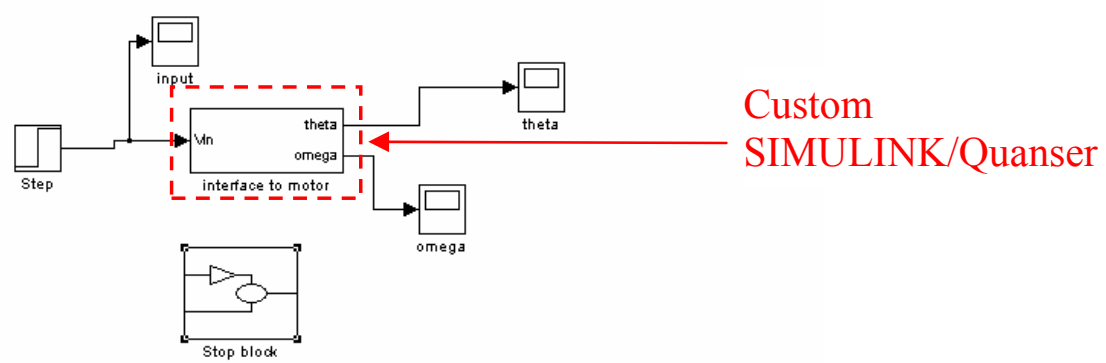

Figure 11: SIMULINK/Quanser for (a) Step Response and (b) Bode

Remark \#4 In order to prevent stiction friction forces from affecting the step response data, the motor was initially rotating at an arbitrary set point speed when the $3.0 \mathrm{~V}$ step change was applied (see Fig. 8).

Remark \#5 Calculation of $K_{t b}$ and $J_{m}$ from step response or frequency response data was not explicitly covered in the procedure but the adjacent Systems Engineering course, ES307 "Linear Systems," covers this topic.

Remark \#6 As with the step response, a bias voltage of $2.0 \mathrm{~V}$ is utilized in the collection of the Bode frequency response data (see Figure 9) in an effort to minimize friction forces at zero velocity.

Remark \#7 Though the automated frequency response block of Figure 9 was utilized for the bulk of data collection, the midshipmen were required to "manually" obtain two frequency response data points. That is, the students were required to apply a sinusoidal voltage input, measure the corresponding output velocity after transients, and then calculate the resulting magnitude difference and phase shift between the input voltage and output velocity signals. This step allows the student to appreciate and understand the automatic collection routine of Figure 9.

\subsection{Combining the Flexible Robotic Link and the DC Motor Model}

With the identification of the flexible robotic link and the DC motor system, the midshipmen were ready to consider the development of the complete system. 


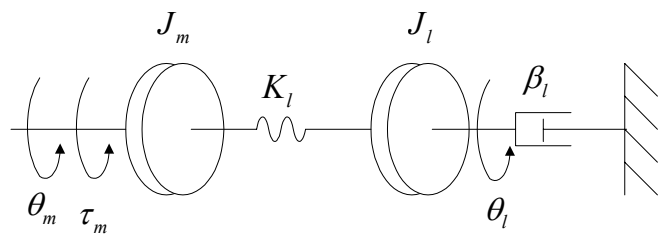

\section{Figure 12: DC Motor and Flexible Link Schematic}

Once the midshipmen produced the correct schematic, the development of the corresponding state space representation was a straightforward process. From Figure 12, the student initially generated two equations of motion from the free body diagram analysis upon which the following state space representation was easily derived

$$
\begin{aligned}
& \dot{\bar{x}}=\left[\begin{array}{cccc}
0 & 1 & 0 & 0 \\
-\frac{K_{l}}{J_{m}} & -\frac{K_{t b}^{2}}{J_{m} R_{a}} & \frac{K_{l}}{J_{m}} & 0 \\
0 & 0 & 0 & 1 \\
\frac{K_{l}}{J_{l}} & 0 & \frac{-K_{l}}{J_{l}} & \frac{-\beta_{l}}{J_{l}}
\end{array}\right] \bar{x}+\left[\begin{array}{c}
0 \\
\frac{K_{t b}}{J_{m} R_{a}} \\
0 \\
0
\end{array}\right] \bar{u} \\
& \bar{y}=\left[\begin{array}{c}
\theta_{m} \\
\theta_{l}
\end{array}\right]=\left[\begin{array}{llll}
1 & 0 & 0 & 0 \\
0 & 0 & 1 & 0
\end{array}\right] \bar{x}+\left[\begin{array}{l}
0 \\
0
\end{array}\right] \bar{u}
\end{aligned}
$$

where the state vector $\bar{x}$ and the control input $\bar{u}$ are defined in the following manner

$$
\bar{x}=\left[\begin{array}{llll}
\theta_{m} & \dot{\theta}_{m} & \theta_{l} & \dot{\theta}_{l}
\end{array}\right]^{T} \quad \bar{u}=\left[e_{a}\right]
$$

Remark \#1 The most difficult concept for the students to grasp is that the torque applied to the robotic link is transmitted through the deflection of the linear spring assembly (see Figure 12).

\subsection{Model Verification}

With the completion of the state space representation, the students were then required to verify $(0.5)$ by applying a step input voltage to both the simulation model and the experimental setup. 


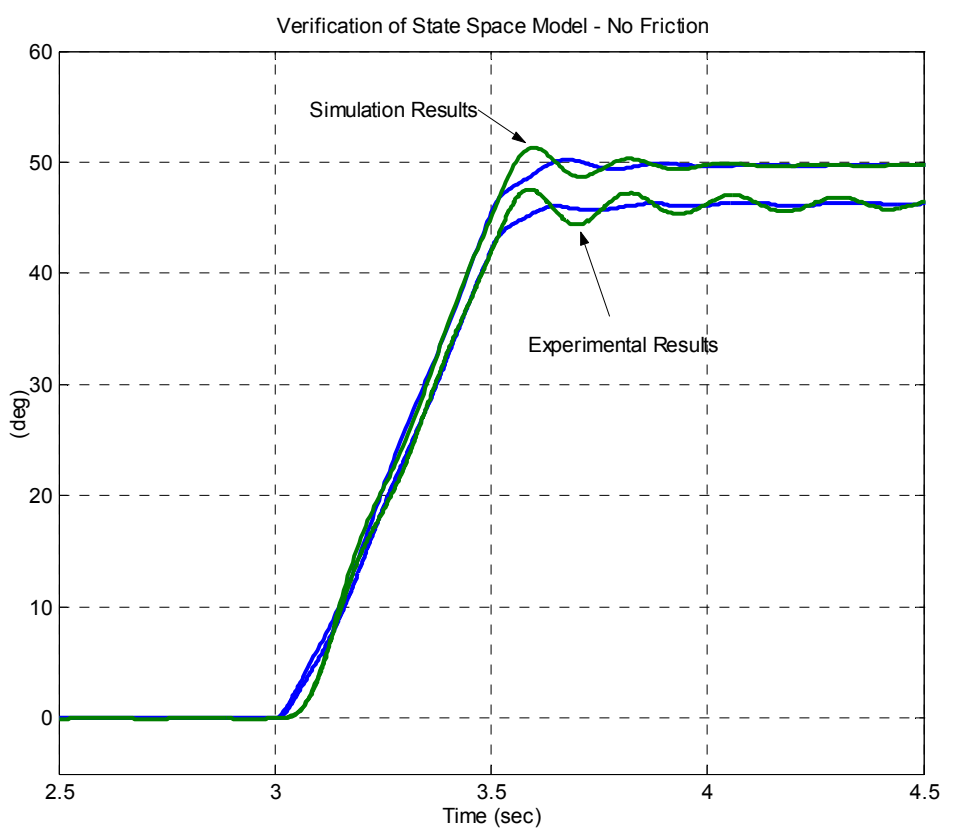

Figure 13: Verification of State Space Representation: the green line represents the angular position of the link and the blue line is the angular position of the motor.

From Figure 13, a significant discrepancy exists between the experimental and simulation results. This difference is due to the nonlinear stiction friction forces that were not considered in the model development. As a result, the students were guided to augment their linear state space representation with a nonlinear coulomb friction input (see Figure 14) in an attempt to capture these frictional effects and approve the overall accuracy of the model. The midshipmen were asked to increase the coulomb friction level until the simulation results approached those obtained by the experiment.

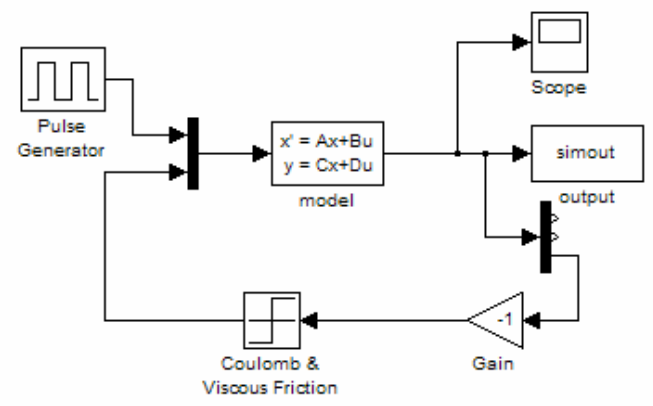

Figure 14: Nonlinear Friction Block Inclusion

Remark \#1 With the inclusion of the friction block, the simulation prediction and experimental measurements correspond as illustrated in Figure 15. 


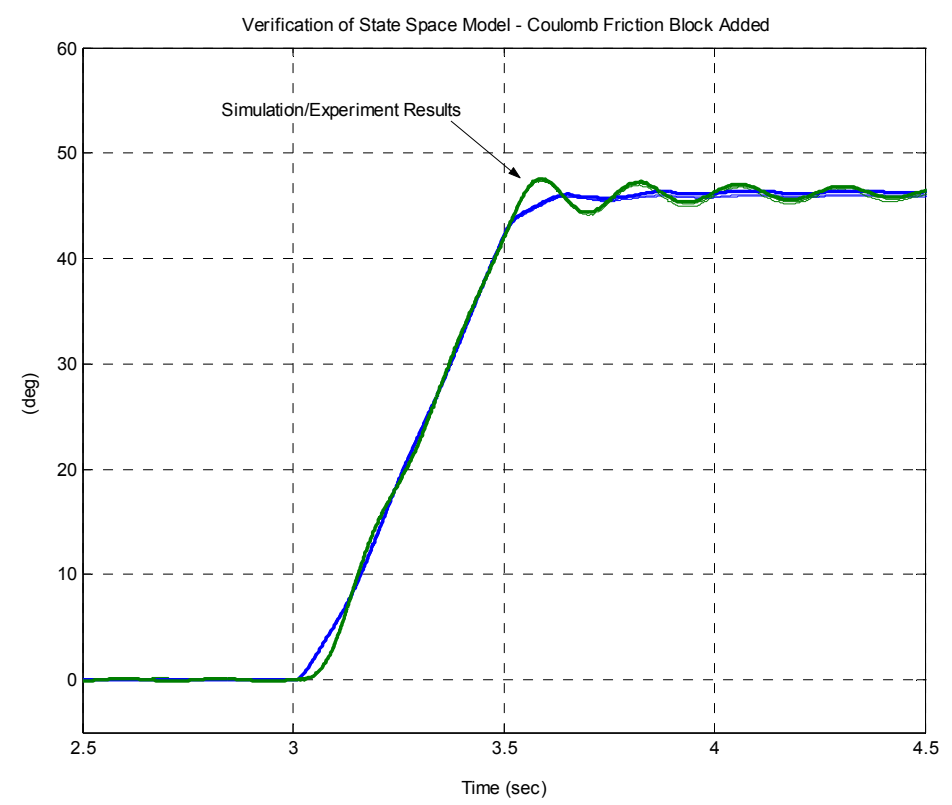

Figure 15: Simulation and Experimental Results with Friction Block

\section{Conclusion}

In an effort to gauge the success of the exercises and to further our educational objectives for the course, an assessment questionnaire was distributed to students in all four sections of the course (taught by different instructors). The questionnaire was unannounced and the responses were anonymous.

\begin{tabular}{|l|c|}
\hline Assessment Questions: (1 = highest score, 5 = lowest score, and N=78) & Average Score \\
\hline 1: Overall opinion of the exercises & 1.35 \\
\hline 2: Did the exercises clarify the modeling procedure? & 1.84 \\
\hline 3: Can you describe the steps in the process? & 1.42 \\
\hline $\begin{array}{l}\text { 4: Did the exercises clarify the goals of the course and reinforce lecture } \\
\text { material? }\end{array}$ & 1.87 \\
\hline $\begin{array}{l}\text { 5: Did the exercise increase your "physical intuition" about the meaning } \\
\text { of various devices and quantities discussed in the course? }\end{array}$ & 2.18 \\
\hline
\end{tabular}

Table 1: Students' Responses to Assessment Questionnaire

Table 1 reports the students' responses to the assessment questionnaire. A score of 1 is the highest and 5 is the lowest. The average score in each category was computed over 78 responses. The overall opinion of the experience was quite high $($ mean $=1.35)$. The response to Question $2($ mean $=$ 1.84) indicates the students' opinion that this sequence of exercises aided in the conceptualization of the over all modeling process. In Question 3, students were asked to sketch out the generic steps in the modeling procedure without consulting their notes. Instructors rated their responses expecting them to generate something resembling Figure 1. The average score (1.42) seems to corroborate the students' responses to Question 2. Question 4 attempts to assess how well the students feel the laboratory experiences were integrated with the remainder of the course. We felt that the score $($ mean $=1.87)$ was an indication that we were successful in that regard. Finally, the midshipmen 
were asked if the procedures aided them in developing insight and intuition about various physical components such as inductors, viscous dampers, etc.; or about the types of parameters encountered in the course such as voltage, moment of inertia, etc. While the mean score of 2.18 was well above the midpoint of the evaluation range of 3 , it is still considerably lower than students' responses to other questions, though the reason is unclear.

In addition to the numerical scores, students were asked for written suggestions and comments. The most common comments, along with our interpretations, follow.

- Many students praised the lab stating that they felt it was a good experience, indicating that it was the type of activity they expected to do in industry. Many enjoyed the opportunity to work with engineering hardware in a hands-on type format.

- Some students felt some of the exercises were too open ended, while others applauded the discovery oriented format. We as instructors agree that there is a delicate balance between providing just enough information for students to succeed and spoon feeding them pre-canned procedures.

- We felt that an added benefit of this exercise was that students gained a more intuitive understanding of reasonable ranges of numerical values for some common engineering parameters. This assisted them later in determining if their results were realistic.

In hindsight, we felt the experiment sequence was a success but intend to make the following changes for the next semester:

- The damping constant of the link, in Section 4.1, proved to be the most difficult parameter to estimate because the damping is nonlinear and very small. An alternative procedure would be desirable.

- For the exercise in Section 4.2 would have the students attempt to evaluate and validate the system ID motor models. In particular, they should evaluate which system identification method produced parameters that would yield a more accurate simulation of the motor behavior. In essence, we need to reinforce the validation process through repetition.

- For the exercise in Section 4.3 we also could have them explicitly account for all gear ratios, internal and external, that were not emphasized within these presented exercises.

- The final laboratory exercise of Section 4.3 could have possibly been split into two lab periods. In particular, the open ended nature of the model refinement step, which involved adding a friction term in the simulation, seemed time consuming. 
[1] Dixon, W., Dawson, D., Costic, B., and de Queiroz, M., "A MATLAB-Based Control Systems Laboratory Experience For Undergraduate Students: Toward Standardization And Shared Resources," IEEE Transactions on Education, Vol. 45, No. 3, pp. 218-226.

[2] Watkins, J. and O’Brien, Jr., R., “A Novel Approach To A Control System Laboratory,” Proceedings of IMECE '03, 2003 ASME International Mechanical Engineering Congress and Exposition, Washington, DC, November 2003.

[3] O’Brien, Jr., R., “On Teaching State-Space Control Systems Design Using A MATLAB RealTime Experiment," Proceedings of the Southeastern Symposium on Systems Theory, Morgantown, WV, March 2003.

[4]Alazard, D. and Chretien, J., "Flexible Joint Control: Robustness Analysis of the Collocated and Non-collocated Feedbacks," Proceedings of IROS'93, Yokohama, Japan, July 1993.

JOEL M. ESPOSITO is an Assistant Professor in the Systems Engineering Department at the United States Naval Academy. He received the M.S. and Ph.D. degree in mechanical engineering from the University of Pennsylvania in 1999 and 2002, respectively. His research interests include algorithmic approaches to testing complex control systems and mobile robotics.

MATTHEW G. FEEMSTER is an Assistant Professor in the Systems Engineering Department at the United States Naval Academy. He received his M.S. and Ph.D. degree in electrical engineering from Clemson University in 1996 and 2000, respectively. His research interests include nonlinear control techniques for such mechatronic systems as electric machinery, robotic manipulator systems, magnetic bearings, and flexible beams/robots/rotors.

JOHN M. WATKINS is an Associate Professor in the Systems Engineering Department at the United States Naval Academy. He received his B.S. degree in electrical engineering from the University of Nebraska-Lincoln in 1989 and his M.S. and Ph.D. degrees in electrical engineering from The Ohio State University in 1991 and 1995, respectively. His research interests include feedback control systems, system identification, fault detection and isolation, magnetic levitation, active noise control, and spacecraft dynamics and control. 